\title{
Simultaneous Vehicle Crossing Effects on Fatigue Damage Equivalence Factors for North American Roadway Bridges
}

\author{
Scott Walbridge, M.ASCE${ }^{1}$; Vincent Fischer ${ }^{2}$; Nariman Maddah ${ }^{3}$; and Alain Nussbaumer ${ }^{4}$
}

\begin{abstract}
In many design codes for roadway bridges, fatigue design involves passing a load model over an influence line for a critical location on the bridge and then determining the resulting nominal stress range. For fatigue design in the finite-life domain, this stress range is then multiplied by a damage equivalence factor to account for differences in the fatigue damage because of the load model and the expected real traffic. In general, the effects of simultaneous vehicle crossings are not considered in the calibration of the damage equivalence factor. In this paper, fatigue design procedures in the U.S., Canadian, European, and Swiss codes applicable for the design of steel or aluminum roadway bridges are first reviewed. A simulation-based study, conducted to investigate the effects of simultaneous vehicle crossings on the damage equivalence factors for North American roadway bridges, is then presented. Based on the results of this study, recommendations are made for amplifying the North American damage equivalence factors in cases where the effects of simultaneous vehicle crossings are expected to be significant. DOI: 10.1061/(ASCE)BE.1943-5592.0000482. (C) 2013 American Society of Civil Engineers.
\end{abstract}

CE Database subject headings: Fatigue; Damage; Steel; Aluminum (material); Highway bridges; North America; Simulation.

Author keywords: Fatigue design; Damage accumulation; Traffic loading; Steel; Aluminum; Roadway bridges.

\section{Introduction}

In many design codes for roadway bridges, fatigue design involves passing a load model over an influence line for a critical location on the bridge and then determining the resulting nominal stress range. For fatigue design in the finite-life domain, this stress range is then multiplied by a damage equivalence factor to account for differences in the fatigue damage because of the load model and the expected real traffic. The bridge codes that employ this approach include (but are not limited to) codes in the United States (AASHTO 2008), Canada [Canadian Standard Association (CSA) 2011a], Europe [European Committee for Standardization (CEN) 2002, 2005, 2006], and Switzerland [Swiss Society of Engineers and Architects (SIA) 2003a, 2003b]. In general, effects of simultaneous vehicle crossings (that is, because of vehicles following each other closely, overtaking, or crossing in opposite directions) are not considered in the calibration of the fatigue damage equivalence factor. This may be appropriate for certain bridge configurations, such as short-span multigirder bridges, where each girder is influenced by only one lane

\footnotetext{
${ }^{1}$ Associate Professor, Civil and Environmental Engineering Dept., Univ. of Waterloo, Waterloo, ON, Canada N2L 3G1 (corresponding author). E-mail: swalbrid@uwaterloo.ca

${ }^{2}$ Master's Student, Steel Structures Laboratory (ICOM), École Polytechnique Fédérale de Lausanne, GC B3 495, Station 18, CH-1015 Lausanne, Switzerland.

${ }^{3}$ Doctoral Candidate, Steel Structures Laboratory (ICOM), École Polytechnique Fédérale de Lausanne, GC B3 495, Station 18, CH-1015 Lausanne, Switzerland. E-mail: nariman.maddah@epfl.ch

${ }^{4}$ Adjunct Professor, Steel Structures Laboratory (ICOM), École Polytechnique Fédérale de Lausanne, GC B3 495, Station 18, CH-1015 Lausanne, Switzerland. E-mail: alain.nussbaumer@epfl.ch

Note. This manuscript was submitted on August 23, 2012; approved on February 12, 2013; published online on February 14, 2013. Discussion period open until May 1, 2014; separate discussions must be submitted for individual papers. This paper is part of the Journal of Bridge Engineering, Vol. 18, No. 12, December 1, 2013. CASCE, ISSN 1084-0702/ 2013/12-1309-1318/\$25.00.
}

of traffic and only one truck is likely to be on the bridge at a time. On the other hand, for structures such as long-span, box girder bridges, the effect of simultaneous crossings on the rate of fatigue damage may be significant. The simultaneous crossing of trucks on these bridges can result in bigger stress cycles, which do more fatigue damage than if the trucks cross separately. This can be explained by the shallow, negative slope of the stress-life (S-N) curve, which can be shown to result in the stress range size having a much greater influence on fatigue damage than the number of stress cycles.

With this in mind, the current paper first reviews the fatigue design procedures in several international codes. A simulationbased study is then conducted to investigate the effects of simultaneous vehicle crossings on the North American damage equivalence factors for fatigue design in the finite-life domain. This study is carried out using software developed by the Steel Structures Laboratory (ICOM) at the École Polytechnique Fédérale de Lausanne (EPFL) in Lausanne, Switzerland, including a traffic simulation program (WinQSIM), which outputs load effect or stress range histograms because of simulated real traffic, and a fatigue damage accumulation program (FDABridge), which uses these histograms to calculate damage equivalence factors. Based on the results of this study, recommendations are made for amplifying the North American damage equivalence factors in cases where the effects of simultaneous vehicle crossings are expected to be significant.

\section{Background}

\section{Fatigue Verification according to North American Codes}

Fatigue verification in AASHTO (2008) consists of verifying that each detail satisfies

$$
\gamma(\Delta f) \leq(\Delta F)_{n}
$$

where $\gamma=0.75$ is the fatigue damage equivalence factor; $\Delta f$ is a live load stress range because of the passage of the design truck; and 
$(\Delta F)_{n}$ is the nominal fatigue resistance. The characteristics of the design truck are reproduced in Fig. 1(a). To these axle weights, a dynamic load allowance (DLA) of $I M=0.15$ (or 0.75 for deck joints) is applied for fatigue verification; that is, the static vehicle load is multiplied by $(1+I M)$. The nominal fatigue resistance is

$$
(\Delta F)_{n}=\left(\frac{A}{N}\right)^{1 / m} \geq 0.5 \cdot(\Delta F)_{T H}
$$

where

$$
N=365 \cdot 75 \cdot n \cdot(A D T T)_{S L}
$$

In Eqs. (2) and (3), $A$ and $m$ are constants defining the vertical position and slope of the design $\mathrm{S}-\mathrm{N}$ curve for the detail category of interest; $n$ is the number of stress cycles per truck passage; $(A D T T)_{S L}$ is the single-lane average daily truck traffic (ADTT); and $(\Delta F)_{T H}$ is the constant amplitude threshold, below which the fatigue life is effectively infinite. The value $n$ is given in a table for a limited number of cases and depends on the location and bridge span.
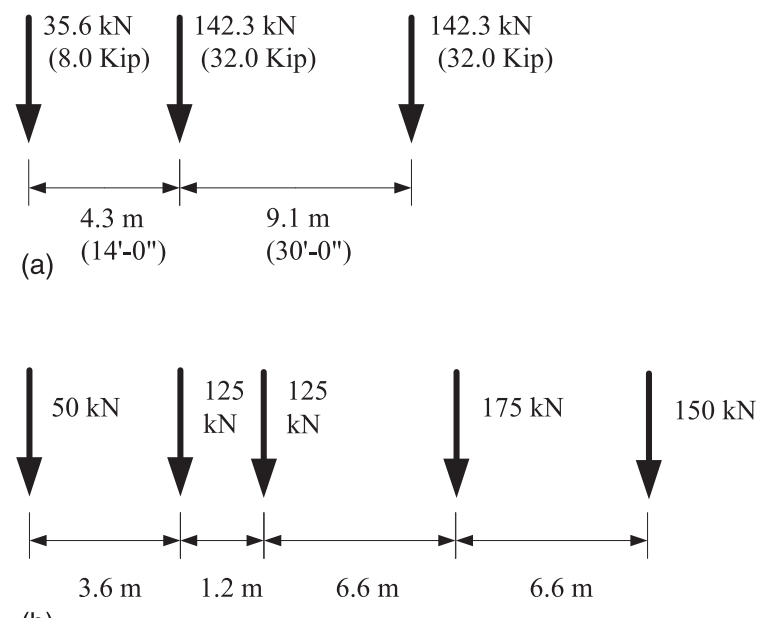

(b)

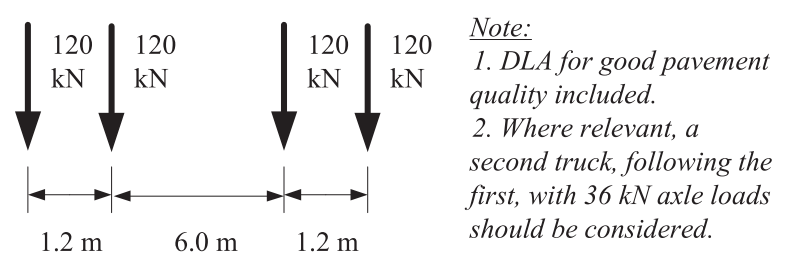

(c)

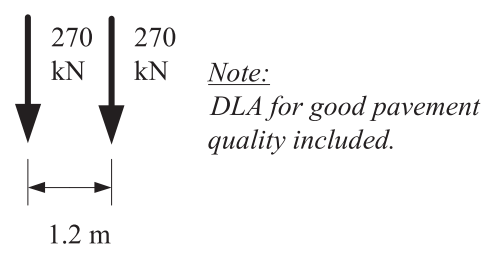

(d)

Fig. 1. Fatigue design trucks used by various codes: (a) AASHTO design truck (data from AASHTO 2008); (b) CAN/CSA-S6 design truck (data from CSA 2011a); (c) CEN 1991-2 fatigue load model (data from CEN 2002); (d) SIA 261 fatigue load model (data from SIA 2003b)


2.0 for spans less than $12.2 \mathrm{~m}(40 \mathrm{ft})$. In the absence of better information, $(A D T T)_{S L}$ is taken as the average daily truck traffic per direction $(A D T T)$ times $p$, where $p$ is taken as $1.0,0.85$, or 0.80 , for one lane, two lanes, and three or more lanes, respectively.

For the fatigue verification of steel structures, the constants $A$ and $(\Delta F)_{T H}$ are tabulated for detail categories from $\mathrm{A}$ to $\mathrm{E}^{\prime}$, and $m$ is assumed to be 3.0 for all detail categories. For the fatigue design of aluminum structures, on the other hand, $m$ varies from 3.42 to 6.45 .

The basis for $\gamma=0.75$ is provided in Moses et al. (1987), where a fatigue design truck is proposed with a gross vehicle weight $(\mathrm{GVW})$ that is 0.75 times that of the code truck for strength design. This factor was determined by taking the GVW histogram from a 27,513-truck survey (Snyder et al. 1985) and calculating an equivalent weight of the fatigue design truck - that is

$$
W_{e q}=\left(\sum f_{i} \cdot W_{i}^{m}\right)^{1 / m}
$$

where $f_{i}=$ frequency associated with $\mathrm{GVW} W_{i}$. This formula is a rearrangement of the well-known Miner's sum; $m=3.0$ is assumed in the calculation of $W_{e q}$.

The fatigue verification in CSA (2011a) bears strong resemblance to the fatigue verification in AASHTO (2008), with the following notable differences:

- A different design truck is employed [see Fig. 1(b)];

- A DLA of 0.25 or more is employed, depending on the number of axles considered;

- A fatigue damage equivalence factor of 0.52 is employed in most cases (0.62 is used for decks) rather than 0.75 [CSA (2011b) provides a justification for this value, which considers differences in the U.S. and Canadian traffic, code trucks, and DLAs]; and

- The S-N curve slopes, $m$, for the aluminum detail categories ranges from 3.45 to 6.85 , based on ADM 2010 (Aluminum Association 2010). Also, for aluminum structures, the fatigue damage equivalence factor varies from 0.54 to 0.65 , depending on the S-N curve slope.

In both codes, $\gamma$ is independent of the bridge span and traffic volume. The fatigue resistance is a function of the detail category and the expected number of cycles during the 75-year service life. For the design of a girder that is affected by loads on several lanes, little guidance is provided. An engineer might choose to design using $A D T T \cdot 1$ (for unidirectional traffic) or $A D T T \cdot 2$ (for bidirectional traffic) instead of $(A D T T)_{S L}$ in Eq. (3). This would account for the effect of the traffic in the other lanes, but not the effect of having multiple trucks on the bridge at the same time.

In a recent study wherein the North American fatigue damage equivalence factors for aluminum were calibrated (Coughlin and Walbridge 2011), the effects of simultaneous vehicle crossings were considered using a relatively simple approach. A model proposed by Nowak (1993) was employed, wherein it is assumed that every 15th truck crosses the bridge side by side with another truck. For every 150th truck, it is assumed that there is a $50 \%$ weight correlation between the two trucks, and for every 450th truck, it is assumed that the two trucks are fully correlated. The remaining trucks are assumed to cross the bridge one at a time. Using this model, simultaneous crossing events were simulated by assuming that the two crossing trucks have their front axles lined up and cross in the same direction. Based on this study, it was recommended that an increase of $\sim 8$ to $20 \%$ in $\gamma$ be considered, for $m=3.0$ to 6.85 .

\section{Fatigue Verification according to European Codes}

Fatigue assessment in CEN $(2002,2005,2006)$ consists of verifying the following: 


$$
\gamma_{F f}\left(\Phi_{2} \cdot \lambda \cdot \Delta \sigma_{F L M}\right) \leq \frac{\Delta \sigma_{c}}{\gamma_{M f}}
$$

where $\gamma_{F f}$ and $\gamma_{M f}=$ partial safety factors for fatigue loading and fatigue strength; $\Phi_{2}=$ dynamic impact factor; $\Delta \sigma_{F L M}=$ stress range because of the fatigue load model [see Fig. 1(c)]; and $\Delta \sigma_{c}=$ a stress range representing the fatigue strength for the detail category. This reference stress range corresponds with the ordinate of the design S-N curve at $N=2 \cdot 10^{6}$ cycles (not at the number of cycles associated with the service life). The effects of traffic volume and service life are contained rather in the damage equivalence factor, $\lambda$, on the load effect side of Eq. (5). This damage equivalence factor is a product of several partial factors, $\lambda_{i}$-that is

$$
\lambda=\lambda_{1} \cdot \lambda_{2} \cdot \lambda_{3} \cdot \lambda_{4} \leq \lambda_{\max }
$$

where $\lambda_{1}$ accounts for the influence line and span effects; $\lambda_{2}$ accounts for the traffic volume; $\lambda_{3}$ accounts for the design life ( $\lambda_{3}=1.0$ for 100 years); and $\lambda_{4}$ accounts for the effect of traffic in lanes other than lane 1 (that is, the slow or far-right-hand lane). $\lambda_{\max }$ accounts for the constant amplitude threshold, below which infinite fatigue life is assumed, similar to the $0.5 \cdot(\Delta F)_{T H}$ term in AASHTO (2008). Thus, $\lambda_{1}, \lambda_{2}$, and $\lambda_{3}$ replace $\gamma$ and $N$ in the North American codes. $\lambda_{1}$ varies linearly from 1.85 to 2.55 for details within the span or from 1.7 to 2.2 according to a two-part linear function for details near supports [see Fig. 2(a)]. $\lambda_{1}$ depends on a critical length, which is equal to the span for simply supported structures, for example. The $\lambda_{4}$ factor, which does not have a counterpart in the North American codes, is calculated as

$$
\lambda_{4}=\left[1+\frac{N_{2}}{N_{1}} \cdot\left(\frac{\eta_{2} \cdot Q_{m 2}}{\eta_{1} \cdot Q_{m 1}}\right)^{5}+\frac{N_{3}}{N_{1}} \cdot\left(\frac{\eta_{3} \cdot Q_{m 3}}{\eta_{1} \cdot Q_{m 1}}\right)^{5}+\cdots\right]^{1 / 5}
$$

where $N_{i}$ is the annual number of trucks in lane $i ; Q_{m i}$ is the equivalent gross weight of the trucks in lane $i$ [which is calculated using Eq. (3) with $m=5$ assumed]; and $\eta_{i}$ is the transverse load distribution factor for lane $i$ (always positive).

In CEN (2005), the S-N curves for most steel details have two slopes, rather than one. Below $N=5 \cdot 10^{6}$ cycles, $m=3.0$. Beyond $N=5 \cdot 10^{6}$ cycles, $m=5.0$. The constant amplitude fatigue limit coincides with the ordinate of the $\mathrm{S}-\mathrm{N}$ curve at $N=5 \cdot 10^{6}$ cycles. Cycles with stress ranges below the ordinate at $1 \cdot 10^{8}$ cycles (that is, the cutoff limit) are ignored.

Fatigue verification in the Swiss codes (SIA 2003a, b) bears strong resemblance to the Eurocode fatigue verification, with the following notable differences:

- A different fatigue load model is employed [see Fig. 1(d)]; and

- The damage equivalence factors, $\lambda_{1}$ and $\lambda_{2}$, have been combined into a single factor, $\lambda_{1}$, which accounts both for bridge geometry and traffic volume effects. This factor is determined by referencing a set of curves, which vary smoothly as a function of the critical length and roadway category [see Fig. 2(b)].

In a recent study by Maddah and Nussbaumer (2012), WinQSIM and FDABridge were used to evaluate the effects of simultaneous
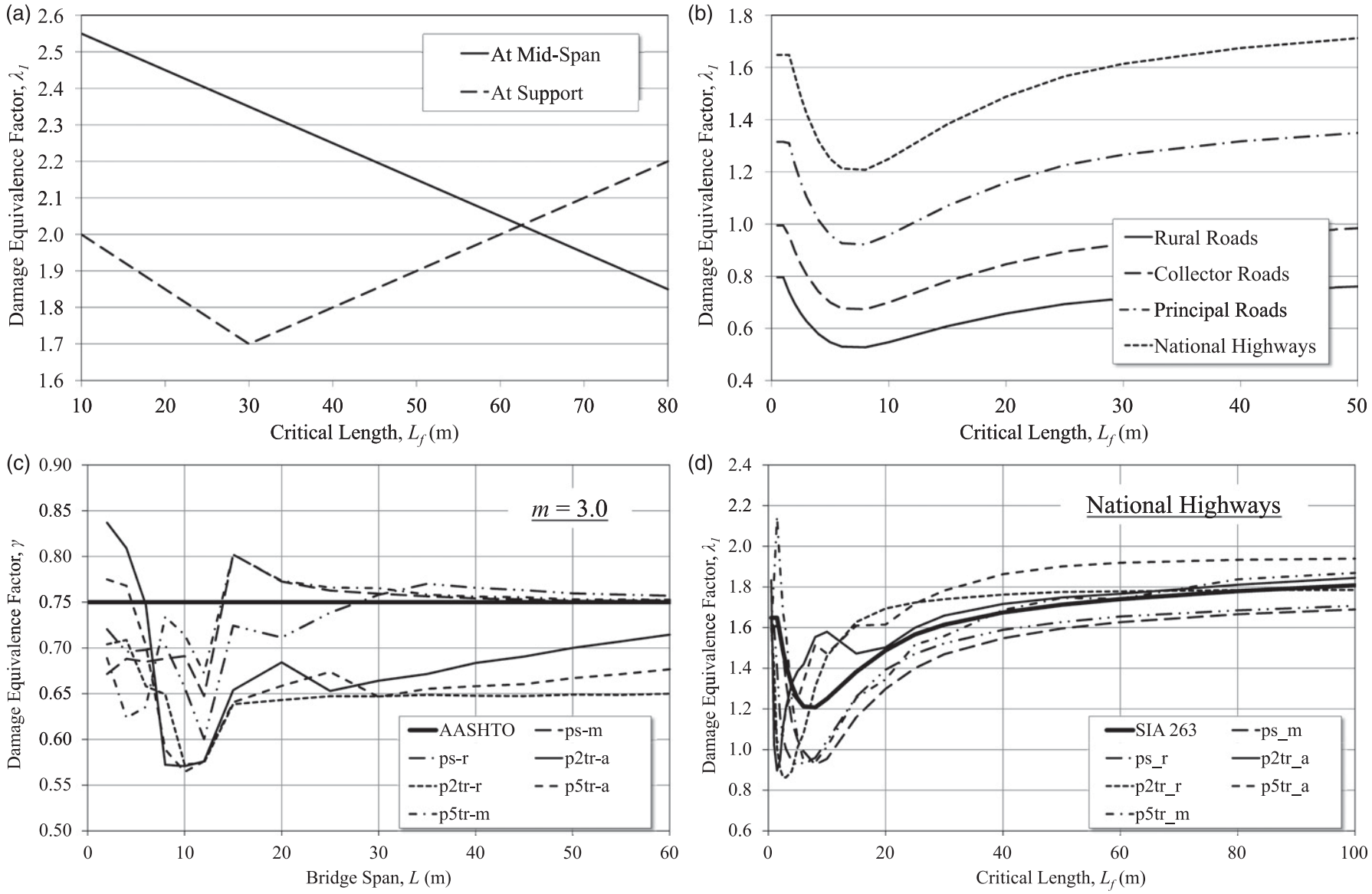

Fig. 2. Damage equivalence factors, $\lambda_{1}$, in (a) EN (2002) and (b) SIA (2003b), and single-crossing simulation results for (c) U.S. traffic and (d) Swiss traffic 
vehicle crossings using Swiss traffic data. As a result of this study, a new single-axle fatigue load model is proposed, in conjunction with a new method for calculating the critical length, based on the area under the influence line. In addition, it is proposed that Eq. (7) be replaced with the following equation:

$$
\begin{aligned}
\lambda_{4}= & {\left[(1-c)+\left(\frac{N_{2}}{N_{1}}-c\right) \cdot\left(\frac{\eta_{2} \cdot Q_{m 2}}{\eta_{1} \cdot Q_{m 1}}\right)^{5}\right.} \\
& \left.+c \cdot\left(1+\frac{\eta_{2} \cdot Q_{m 2}}{\eta_{1} \cdot Q_{m 1}}\right)^{5}\right]^{1 / 5}
\end{aligned}
$$

where $c=$ simultaneous crossing rate, calculated as the number of simultaneous crossings divided by the traffic volume in lane 1 . Eq. (8) is derived based on Miner's sum, and assuming that the stress ranges in the two lanes can be added during simultaneous crossings.

Based on the analysis of the Swiss traffic, a maximum crossing rate of $c=20 \%$ is proposed for bidirectional highway traffic. For unidirectional two-lane highway traffic, a maximum crossing rate of $c=2.5 \%$ is proposed when $20 \%$ of the trucks are in lane 2 . It should be noted that $c$ is an effective crossing rate, which accounts for the effects of interlane simultaneous crossings because of vehicles overtaking (either side by side or staggered) or crossing in opposite directions.

\section{Effects of Influence Line and Bridge Span on Fatigue Damage Equivalence Factors}

In calibrating the damage equivalence factors in SIA (2003a, b) and Coughlin and Walbridge (2011), influence line and bridge span effects were seen to introduce a high degree of scatter. This is shown in Figs. 2(c and d) for two illustrative cases where the simulation results are compared with code values for several influence lines (plotted in Fig. 3). Although the design provisions in the various codes differ in many respects, it is worth noting that no code has been entirely successful in eliminating this important source of scatter.

\section{Parametric Study of Simultaneous Vehicle Crossing Effects}

Given this knowledge of the North American and European code provisions, the main goal of the current study was to assess the


Fig. 3. Influence lines for different locations on (a) one-span; (b) twospan; and (c) five-span bridges effects of simultaneous vehicle crossings on the North American damage equivalence factors for fatigue design in the finite-life domain. In the following paragraphs, a parametric simulation-based study carried out to achieve this goal is described.

\section{WinQSIM and FDABridge Software Overview}

The WinQSIM software, written in the programming language C\#, is based on the probabilistic traffic model developed by Bailey (1996). Its development is further discussed in Meystre and Hirt (2006). The required inputs include a probabilistic traffic model, the number of traffic lanes and the travel direction for each lane, influence lines for each lane, percentages of the total traffic and trucks in each lane, and the traffic volume $(V)$ and speed $(s)$.

The probabilistic traffic model consists of a (user-defined) number of truck types, along with beta distributions for the GVWs, axle weights, and spacings for each truck type. The traffic modeling consists of a Monte Carlo simulation, where parameters for each successive truck are chosen and the vehicle positions are shifted in a stepwise manner. Stress cycles are counted and recorded in a stress range histogram using the rainflow cycle counting method.

Both congested and free-moving conditions can be modeled. The distance between vehicles for the free-moving traffic condition is given by a shifted exponential probability distribution (Bailey 1996). The probability density function (PDF) for this distribution is as follows:

$$
f_{D}(d)=\frac{V}{3600 \cdot s} \cdot \exp \left[-\frac{V}{3600 \cdot s} \cdot(d-5.5)\right]
$$

where $V=$ traffic volume in vehicles per hour; $s=$ traffic speed in meters per second; and $d=$ distance between vehicles in meters. This approach assumes full independence of the spacing between subsequent vehicles. Nontruck traffic can be included in the analysis. The stress ranges because of automobiles are assumed to be negligible. Thus, the main effect of automobiles is to influence the spacing between the trucks. The program can be run with or without a graphical display of the vehicles passing over the bridge (see Fig. 4).

The FDABridge program requires as input the following: a realtraffic stress range histogram, the fatigue load model geometry, and the shape of the design S-N curve. The damage equivalence factor, $\lambda$, is then obtained by shifting the S-N curve vertically until the cumulative damage index based on Miner's sum equals 1.0 for the real-traffic stress range histogram. $\lambda$ is then calculated by dividing the stress range at $2 \cdot 10^{6}$ cycles for this S-N curve by the stress range because of the passage of the fatigue load model (Hirt et al. 2006). For calculation of the North American $\gamma$ factors using the FDABridge software, the following conversion formula is needed:

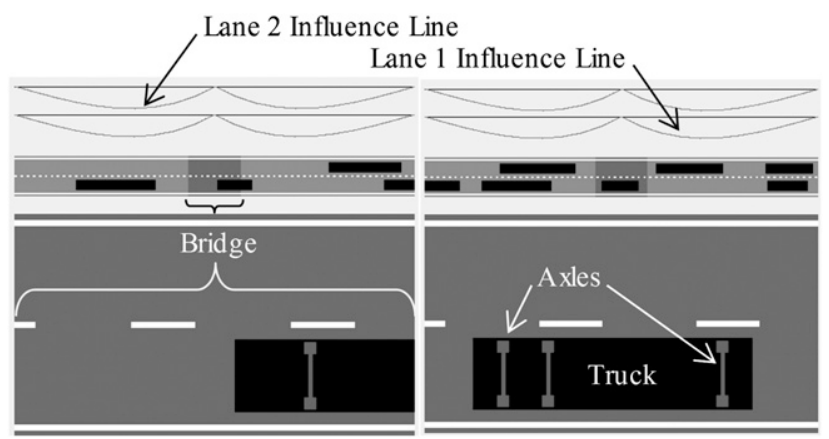

Fig. 4. Graphical display from WinQSIM traffic simulation program 


$$
\gamma=\frac{\lambda}{\left(\frac{n \cdot N_{t r, \mathrm{sim}}}{2 \cdot 10^{6}}\right)^{1 / m}}
$$

where $N_{t r, \text { sim }}=$ number of trucks passing over the bridge during the simulation period. This factor accounts for the fact that the North American codes employ a fatigue strength based on the expected number of cycles during the service life, rather than a characteristic stress range at $2 \cdot 10^{6}$ cycles. As discussed in Coughlin and Walbridge (2011), the $\gamma$ factor is independent of $N$ if the design $\mathrm{S}-\mathrm{N}$ curve has only one slope. A large enough analysis period is needed though to obtain a representative sample of the real-traffic damage. On this basis, $N_{t r \text { sim }}=2 \cdot 10^{6}$ was used in the simulations performed for the current study. Similarly, the DLA has no influence on $\gamma$, if it is assumed to be the same for both the fatigue design truck and the real traffic.

\section{Probabilistic Traffic Models}

The truck databases used to construct the probabilistic traffic models are the same ones used to calibrate the $\gamma$ factors in the current U.S. and Canadian codes. Specifically, the database from Snyder et al. (1985) was used to model the U.S. traffic, and a 10,198-truck database, based on weigh station measurements conducted throughout the province of Ontario in 1995, was used to model the Canadian traffic (CSA 2007). These databases are now arguably outdated. However, the authors are aware of no newer publicly available databases as large (in terms of size and regional coverage) and containing similar axle weight and spacing data. Their use also makes it possible to validate the results by comparison with the current $\gamma$ factors.

The Snyder et al. (1985) database included 27,513 trucks. Of these, 25,901 could be categorized into one of 11 truck types (see Table 1). The database contains only GVW histograms for each truck type. Thus, the axle spacing and distribution of weight between each axle had to be treated as deterministic parameters. The percentages of each truck type in the database and the GVW histograms for each truck type were known, on the other hand, and could be used to select the truck type and GVW probabilistically in the Monte Carlo simulation.

The CSA (2007) database included axle weight and spacing data for each measured truck. Thus, it was possible to model these parameters probabilistically for the Canadian traffic simulations. To define the probabilistic traffic model, a set of truck types must be established. As a starting point, the same 11 truck types as defined by
Snyder et al. (1985) were used, and ranges for each axle weight and spacing were defined, so that trucks could be slotted into one of these truck types. Care had to be taken in the establishment of these ranges, so that a truck could not be slotted into more than one type. Using this approach, a significant number of trucks remained uncategorized. Thus a number of additional truck types were added, with the goal of increasing the number of classified trucks to at least $90 \%$ of the total database. In the end, one 3 -axle, three 4-axle, ten 7-axle, and ten 8-axle truck types were added. Even though the 7 - and 8 -axle trucks comprised less than $15 \%$ of the database, a large number of truck types were needed to categorize them because of the diversity in the axle configurations [see Fischer (2012) for further details]. For illustrative purposes, Fig. 5 shows samples of the statistical analysis performed for one truck type to establish the probabilistic traffic model.

\section{Traffic Flow Modeling}

Traffic flow rates on bridges can be highly site and time dependent. Because the goal of the study was to come up with a generic model for considering simultaneous vehicle crossing effects, the flow conditions were initially varied over a wide range, to identify worst-case scenarios.

The only influence of nontruck traffic (that is, automobiles) on the analysis results was seen to be an increase in the spacing between the trucks. This effect was seen in preliminary studies to be similar to reducing the flow rate in a truck-only simulation. The percentage of trucks in the traffic is also highly site and time dependent. Thus, to produce generic results, the simulations were performed by simulating truck-only traffic. The reported flow rates are therefore truck flow rates. These rates are varied over a wide range, to capture the peak damage equivalence factor. The following truck flow rates were modeled: $0.047,0.1,0.2,0.5,0.75$, and 1.0 vehicles/s. (Note: The flow rates in this paper are all per lane.) The lowest flow rate was chosen to ensure no simultaneous crossings for a one-lane bridge with a length of $460 \mathrm{~m}$ (the maximum investigated bridge length). A practical upper bound on the flow rate for freeways is 0.67 vehicles/s (2,400 vehicles/h) according to Transportation Research Board (TRB 2010). Simulations under the higher flow rates were performed, however, to make sure that peak $\gamma$ values were captured. The vehicle speed, $s$, was set to $22 \mathrm{~m} / \mathrm{s}(80 \mathrm{~km} / \mathrm{h})$ in all simulations. WinQSIM is not able to model traffic flow with variable speed. Thus, it does not model overtaking or lane changes within the bridge length.

Table 1. Truck Types Used in U.S. and Canadian Probabilistic Traffic Models

\begin{tabular}{|c|c|c|c|c|c|c|c|c|c|c|}
\hline \multirow[b]{2}{*}{ Truck category } & \multirow[b]{2}{*}{ Truck type } & \multicolumn{4}{|c|}{ Axle load $(\%)$} & \multicolumn{3}{|c|}{ Axlespacing $[\mathrm{m}(\mathrm{ft})]$} & \multicolumn{2}{|c|}{ Trucks $(\%)$} \\
\hline & & 1 & 2 & 3 & 4 & 1 & 2 & 3 & United States & Ontario \\
\hline Two-axle singles & SU2 & 40 & 60 & - & - & $4.88(16)$ & - & - & 12.9 & 15.6 \\
\hline Three-axle singles & $\begin{array}{l}\text { SU3 } \\
\text { SU4 }\end{array}$ & 30 & 70 & - & - & $5.49(18)$ & - & - & 6.7 & $\begin{array}{l}4.7 \\
0.1\end{array}$ \\
\hline Two-axle semitrailers & 2-S1 & 27 & 40 & 33 & - & $3.66(12)$ & $9.76(32)$ & - & 3.2 & 0.3 \\
\hline Three-axle semitrailers & $\begin{array}{l}2-\mathrm{S} 2 \\
3-\mathrm{S} 1\end{array}$ & 23 & 35 & 42 & - & $3.66(12)$ & $8.54(28)$ & - & 11.1 & $\begin{array}{l}0.9 \\
0.2\end{array}$ \\
\hline Four-axle semitrailers & $\begin{array}{l}3-\mathrm{S} 2 \\
2-\mathrm{S} 3 \\
3-\mathrm{S} 3\end{array}$ & 18 & 45 & 37 & - & $4.27(14)$ & $9.76(32)$ & - & 62.1 & $\begin{array}{r}44.9 \\
0.2 \\
16.3\end{array}$ \\
\hline Five-axle/six-axle semitrailers & $\begin{array}{l}2-\mathrm{S} 1-2 \\
3-\mathrm{S} 1-2\end{array}$ & 17 & 29 & 42 & 12 & $3.05(10)$ & $7.62(25)$ & $7.62(25)$ & 4.0 & $\begin{array}{l}0.5 \\
0.5\end{array}$ \\
\hline Other & - & - & - & - & - & - & - & - & 0.0 & 15.8 \\
\hline
\end{tabular}

Note: Axle load and spacing values are deterministic values used in U.S. traffic simulations. 

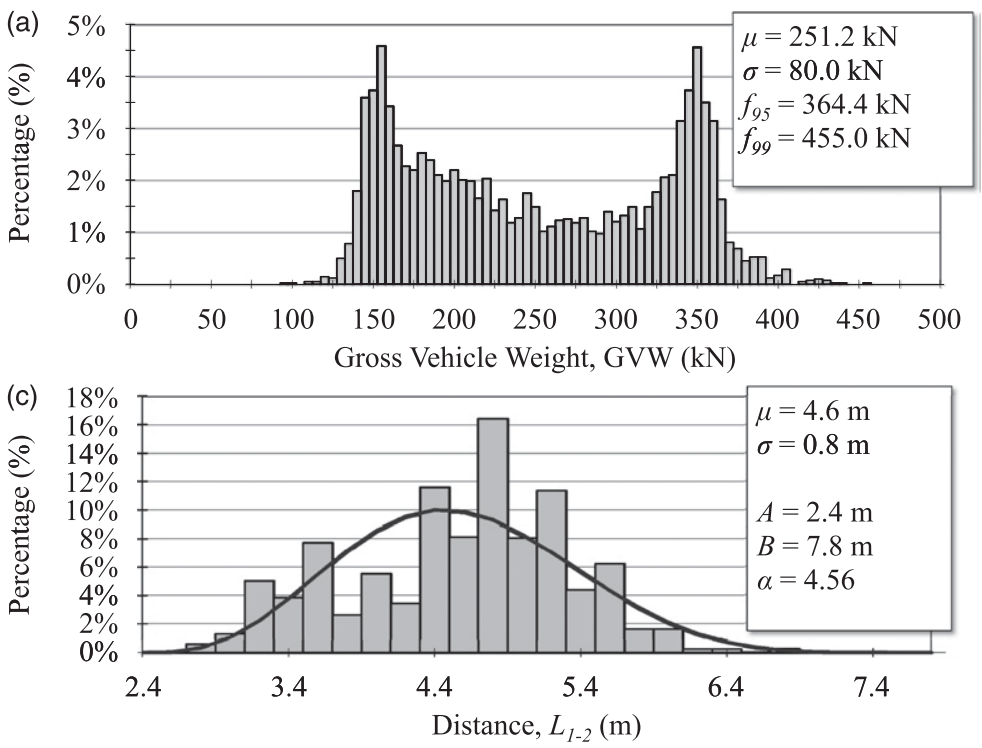

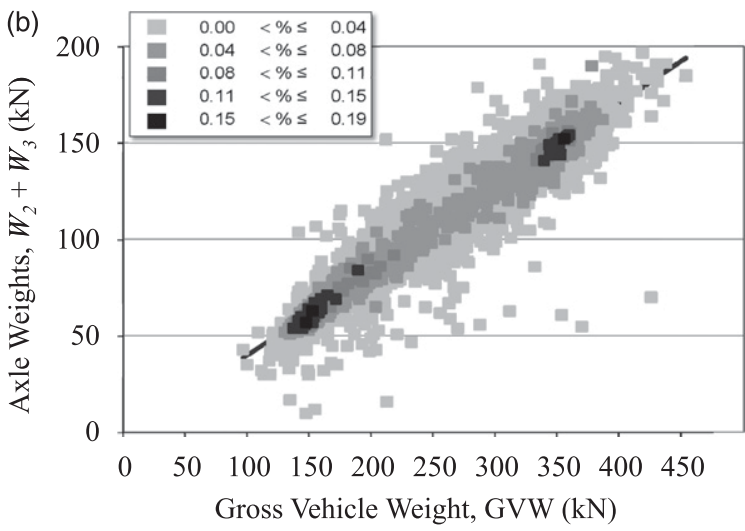

(d)

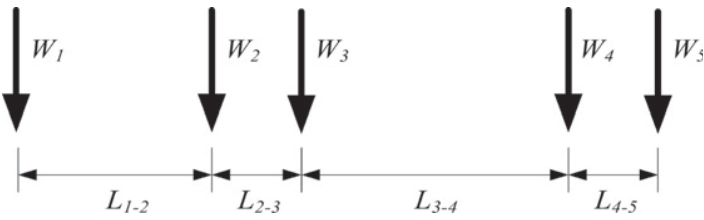

Fig. 5. Sample of statistical analysis of data from CSA (2007) database for 3-S2 truck: (a) GVW histogram; (b) axle weight correlation analysis; (c) fitting of beta distribution to axle spacing; (d) axle configuration

\section{Bridge Modeling}

To model a bridge in WinQSIM, influence lines are needed for each traffic lane. For the current study, the following cases were investigated (Fig. 3): bending moment at the midspan for a simple span girder (ps-m), a two-span girder (p2tr-m), and a five-span girder (p5tr-m); support reaction for a simple span girder (ps-r); and negative bending moment at the midsupport for a two-span girder (p2tr-a). The following spans were considered: 15, 25, 50, and $100 \mathrm{~m}$. For the five-span girder, the outer spans lengths were $80 \%$ of the interior spans.

The following lane configurations where investigated: a onelane bridge, a two-lane bridge with unidirectional traffic, and a two-lane bridge with bidirectional traffic. For the two-lane unidirectional bridge, a worst-case scenario of $50 \%$ of traffic in each lane was modeled. In addition, it was conservatively assumed that the truck GVW histograms are the same in both lanes, even though it is recognized that heavier trucks tend to travel in lane 1 (O'Brien and Enright 2011). For the two-lane bidirectional bridge, equal traffic volumes were assumed in each direction.

The contribution of each traffic lane to the fatigue damage depends on the degree to which the bridge is capable of redistributing loads transversally. For this study, two extreme cases were considered. In the case of a multigirder bridge with a flexible deck, each girder will be influenced by only one lane of traffic. Thus, this case is modeled using the single-lane bridge analysis. The other extreme would be a very stiff single box girder bridge. In this case, both lanes would influence the fatigue verification equally-that is, $\eta_{1}=\eta_{2}=1.0$. This case is therefore modeled by assuming that the same influence line applies to both traffic lanes.

\section{S-N Curves}

The analyses for the current study were performed assuming an S-N curve with a single slope of either $m=3.0$ (for steel) or $m=6.85$ (the largest $m$ value for aluminum)

\section{Parametric Study Results}

Key trends observed in the parametric study output are illustrated in Figs. 6 and 7, using the U.S. $\gamma$ factor results for illustrative purposes. Fig. 6 shows $\gamma$ versus truck flow rate curves for two influence lines (ps-m and p2tr-a) and the four investigated spans. In Fig. 6(a), results for the one-lane analysis are plotted. These actually show $\gamma$ decreasing with an increase in the flow rate for the 15- to 50-m spans. The reason for this trend is that simultaneous crossings happen on a one-lane bridge only if the following truck arrives on the bridge before the leading truck leaves. This may increase the maximum stress. However, it also decreases the minimum stress, because the stress does not drop down to zero between trucks. The relative importance of these two effects determines whether $\gamma$ increases as a result of the simultaneous vehicle crossings.

Fig. 6(b) shows similar results for the bidirectional analysis. In this case, there is a possibility of having two trucks near the midspan in adjacent lanes. As a result, the peak $\gamma$ values are higher. In general, they increase, up to a truck flow rate of $\sim 0.5$ vehicles/s, after which, they decrease. In Fig. 6(c), results for the p2tr-a influence line show similar trends, but with much higher peak $\gamma$ values. The reason for this is that the p2tr-a influence line has a parabolic (rather than triangular) shape, and two peaks (rather than one). Thus, there is a greater chance of two trucks being at locations along the bridge length associated with high stress levels.

Looking at the $\gamma$ values in Fig. 6, it can be seen that for the cases where simultaneous crossing effects are small, the results are very close to the code value of 0.75 . Results below 0.75 are seen in some cases for the p2tr-a influence line. This can be explained by the fact that $n$ steps up to 1.5 for this influence line, whereas $n=1.0$ otherwise. The highest observed damage equivalence factor was 1.17 for a $100-\mathrm{m}$-long bridge, p2tr-a influence line, and flow rate $=0.5$ vehicles $/ \mathrm{s}$. It should be noted that the results of the two-lane unidirectional analysis, with $50 \%$ of the total traffic in each lane, were very similar to the two-lane bidirectional results, and are therefore not shown. It is expected that these results would fall between the results presented in Fig. 6 for one-lane and bidirectional two-lane traffic if a more realistic volume split between lanes were assumed.

It should also be noted that the $\gamma$ values for the two-lane bidirectional case are calculated with $N$ based on ADTT 2 instead of $(A D T T)_{S L}$, as would normally be done for a single box girder bridge. Thus, the increase in $\gamma$ in this figure is solely because of the effects of 

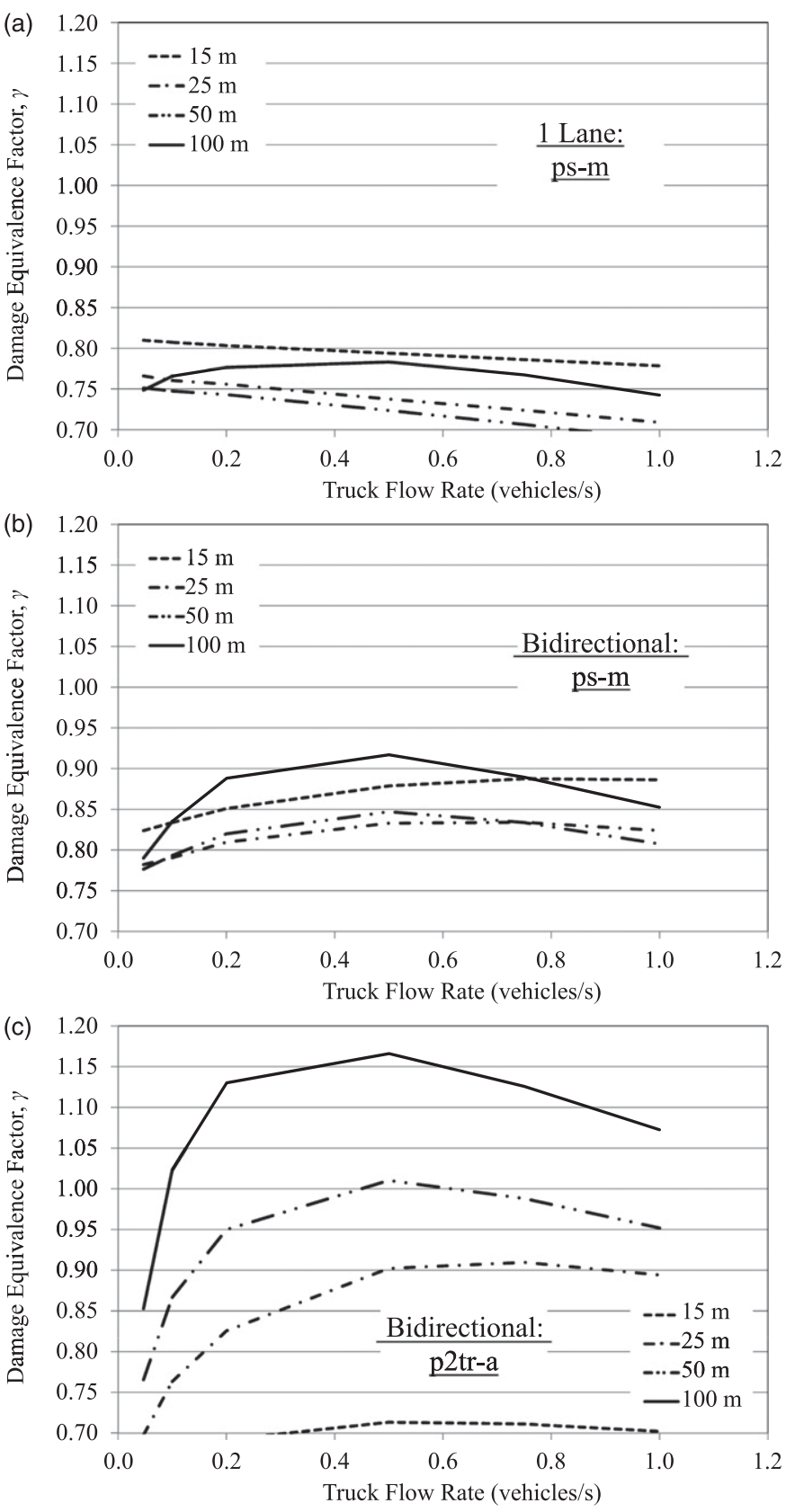

Fig. 6. U.S. traffic simulation results for (a) one-lane bridge, ps-m influence line; (b) bidirectional traffic, ps-m influence line; (c) bidirectional traffic, p2tr-a influence line

simultaneous vehicle crossings, and not simply because a second traffic lane has been added.

\section{Discussion}

Field measurements of traffic flow rate versus speed tend to show a common trend. As illustrated in Fig. 7(a), the flow rate is the greatest at a speed $S_{0}$, which is less than the maximum speed, $S_{f}$. According to the Highway capacity manual (TRB 2010), a practical limit on the flow rate for two-lane highways is $V_{m}=1,700 \mathrm{epc} / \mathrm{h}$, where epc $=$ equivalent passenger cars. $V_{m}=2,400 \mathrm{epc} / \mathrm{h}$ is a practical limit for freeways. Trucks and buses have a passenger car equivalent (PCE) ranging from 1.5 to 4.5 , depending on the terrain. Assuming a typical value of 2.5 , a relationship can be plotted between truck percentage and maximum flow. As shown in Fig. 7(b), this relationship flattens out as the traffic composition approaches $100 \%$ trucks to a value ranging from 0.19 to 0.27 vehicles/s. Although real-traffic compositions can vary considerably, this number is normally well below $100 \%$ trucks. According to TRB (2010), values of 5 or $12 \%$ are typical for urban and rural roads, and values greater than $25 \%$ are considered extreme.

On this basis, the analyzed truck flow rate of 0.2 vehicles/s is considered in the current study to be representative of a flow rate at the high end of the plausible range for quantifying the severity of simultaneous truck crossing effects. Analysis results for the U.S. traffic simulations are thus plotted in Fig. 8, with truck flow rates below or equal to 0.2 vehicles/s used to calculate the average and maximum $\gamma$ curves. Looking at this figure, it can be seen that average $\gamma$ values as high as 0.81 or 0.92 are predicted for the one-lane and two-lane bidirectional cases, for the 0.2 vehicles/s truck flow rate. Curves are also plotted in Fig. 8, with $\gamma$ divided by the current code value of 0.75 . The resulting value is essentially an amplification factor $\left(\gamma_{2}\right)$ that can be used to consider the effects of simultaneous vehicle crossings. This factor can be as high as 1.08 or 1.22 for the one-lane and two-lane bidirectional cases, for the 0.2 vehicles/s truck flow rate. Also shown in Fig. 8 is a thick solid line, corresponding to the following equation:

$$
\begin{aligned}
\gamma_{2}=\{[ & (1-c)+\left(\frac{N_{2}}{N_{1}}-c\right) \cdot\left(\frac{\Delta f_{2}}{\Delta f_{1}}\right)^{m} \\
& \left.\left.+c \cdot\left(1+\frac{\Delta f_{2}}{\Delta f_{1}}\right)^{m}\right] \cdot\left(\frac{N_{1}}{N_{1}+N_{2}}\right)\right\}^{1 / m}
\end{aligned}
$$

with the simultaneous crossing rate varying linearly from $c=0 \%$ at a bridge span of zero, to $c=4.6 \%$ for the one-lane case or $13.5 \%$ for the two-lane case at a bridge span of $100 \mathrm{~m} . \Delta f_{1} / \Delta f_{2}$ and $N_{1} / N_{2}$ are the design stress ranges and traffic volumes associated with the two load sources - that is, the trucks in lanes 1 and 2 for two-lane bridges or the leading and following trucks in a one-lane bridge. In the second case, $\Delta f_{1} / \Delta f_{2}$ and $N_{1} / N_{2}$ are assumed to be equal.

Eq. (11) is simply Eq. (8), modified so that (1) the S-N curve slope, $m$, can be varied; and (2) it is considered that the $\gamma$ values in the current study were calculated using $A D T T \cdot 1$ (for unidirectional traffic) or $A D T T \cdot 2$ (for bidirectional traffic) instead of $(A D T T)_{S L}$. [Note: If $\gamma$ is calculated based on $(A D T T)_{S L}$, then the $N_{1} /\left(N_{1}+N_{2}\right)$ term can be removed.] For the special case where the traffic volumes and stress ranges associated with the two load sources are equal, Eq. (11) simplifies to

$$
\gamma_{2}=\left[(1-c)+0.5 \cdot c \cdot 2^{m}\right]^{1 / m}
$$

The assumed values selected for $c$ are effective simultaneous crossing rates, established by trial and error with the goal of bounding the mean curves for the 0.2 vehicles/s flow rate.

Fig. 9 shows a similar set of amplification factor results based on the Canadian traffic simulations. Results are shown for $m=3.0$ and for $m=6.85$ (the maximum value for aluminum). For plotting these curves, $\gamma=0.52$ was assumed for $m=3.0$ and $\gamma=0.65$ was assumed for $m=6.85$. These results show that the assumed simultaneous crossing rates also give good predictions of the average 0.2 vehicles/s amplification factor for the Canadian traffic simulations. They also show that Eqs. (11) and (12) can be extended to S-N curve slopes other than $m=3.0$. The peak values of $\gamma_{2}$ in this figure are 1.20 and 1.54 for $m=3.0$ and 6.85 .

In the previous analyses, the DLA was assumed to be constant and equal to the code-specified value for both the fatigue design truck and the real traffic. The actual dynamic response of a bridge 

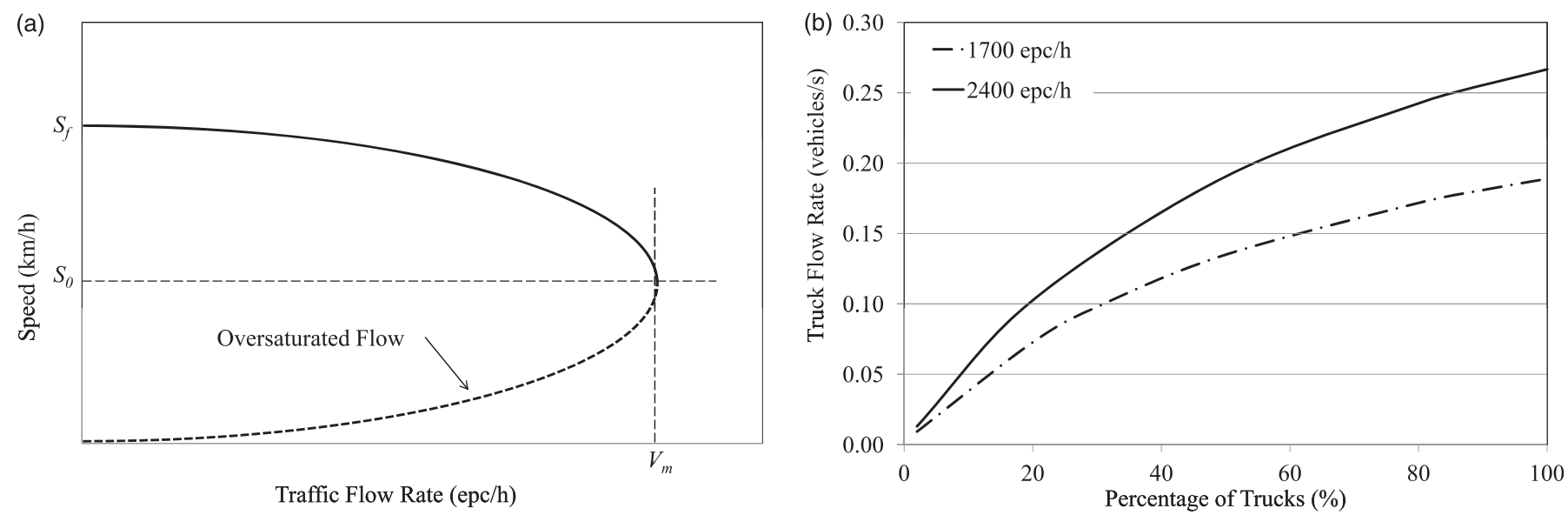

Fig. 7. Relationships between (a) traffic speed and flow rate and (b) truck percentage and flow rate based on TRB (2010)


Fig. 8. U.S. traffic simulation results for flow rates $\leq 0.2$ vehicles/s: (a) $\gamma$ from one-lane analysis; (b) $\gamma_{2}$ from one-lane analysis; (c) $\gamma$ from bidirectional analysis; (d) $\gamma_{2}$ from bidirectional analysis

as trucks pass over it depends on many parameters, including the number of trucks on the bridge, their weight(s), their speed(s), the bridge stiffness, and the road surface quality. The presented analyses may overestimate the effects of simultaneous vehicle crossings on fatigue damage because, as noted in the commentary of AASHTO (2008) and shown in several studies (e.g., Bailey 1996; Maclean and March 1998; Ludescher 2003), the DLA generally decreases with an increase in truck weight and is lower for simultaneous truck crossing events, because the dynamic effects of the multiple trucks will likely be out of phase.

To further study this the DLA influence, several of the simulations were repeated with the code $I M$ applied to the fatigue design truck and an $I M$ from Ludescher (2003) applied to the real traffic, which varies as a function of the total static live load acting on the bridge at any point in time. This model and sample analysis results are presented in Fig. 10. 

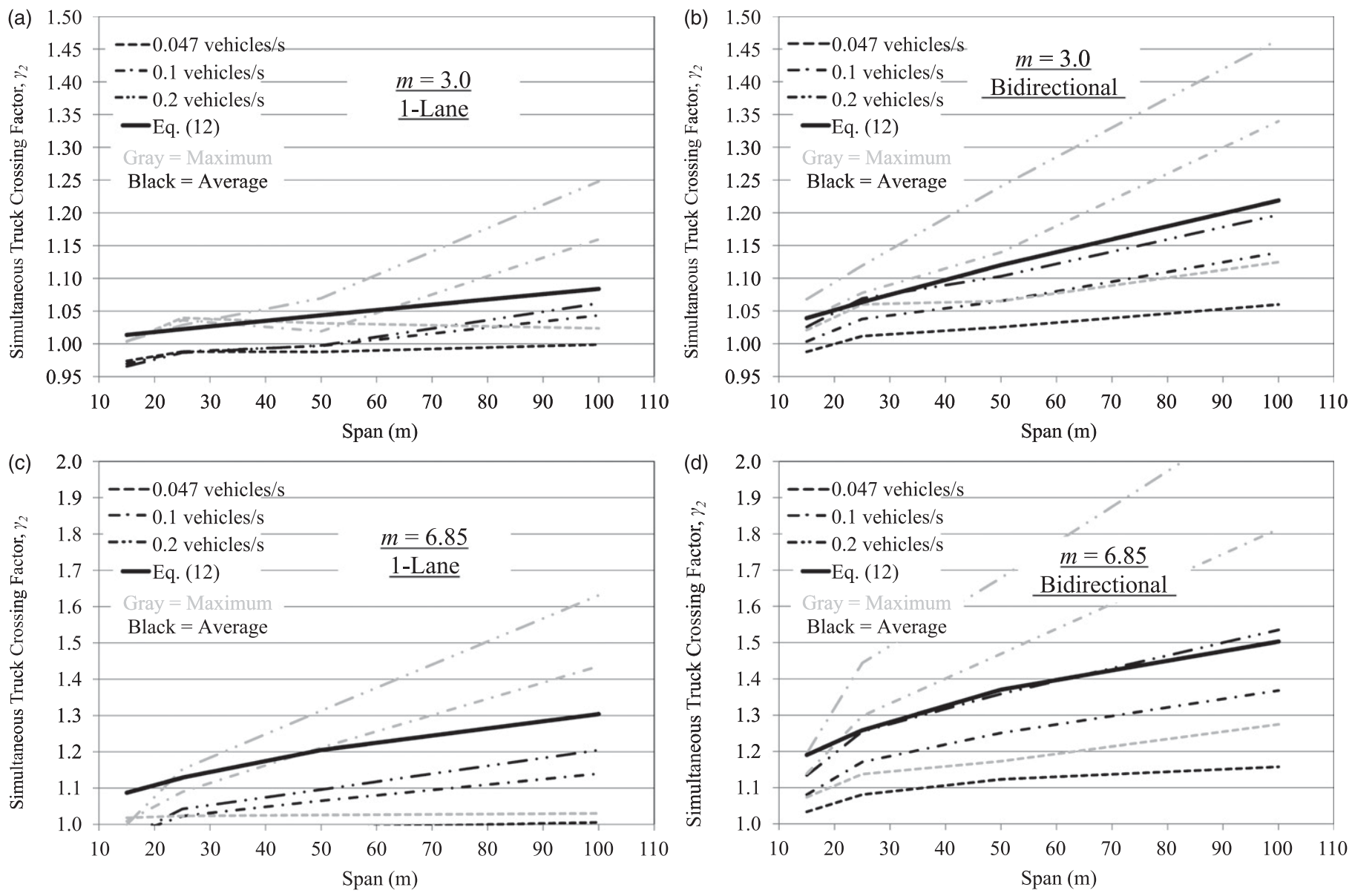

Fig. 9. Canadian traffic simulation $\gamma_{2}$ results for flow rates $\leq 0.2$ vehicles $/ \mathrm{s}$ : (a) one-lane $m=3.0$; (b) bidirectional $m=3.0$; (c) one-lane $m=6.85$; (d) bidirectional $m=6.85$
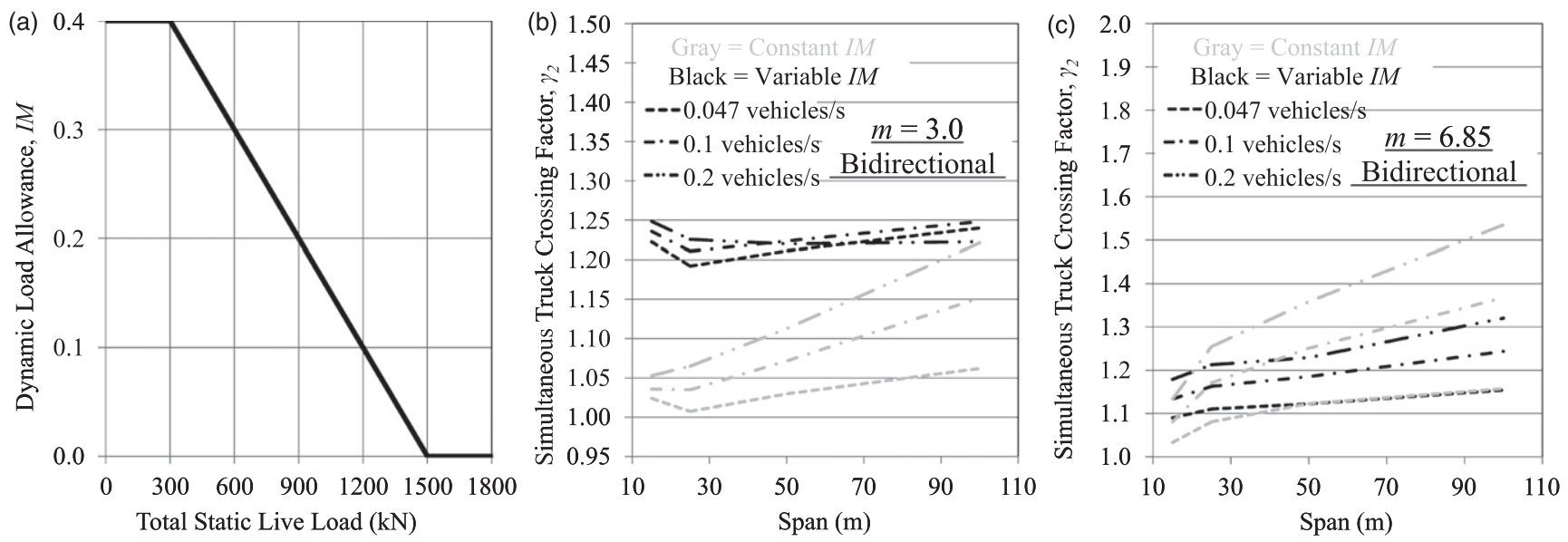

Fig. 10. $I M$ analyses and comparisons: (a) variable $I M$ model from Ludescher (2003); (b) U.S. traffic simulation; (c) Canadian traffic simulation average $\gamma_{2}$ results

Looking at this figure, it can be seen that the effect of the variable $I M$ is to reduce the slopes of the $\gamma_{2}$ versus span curves and to reduce the spread between these curves for different flow rates. At the lower spans (less than $30 \mathrm{~m}$ ), the curves are shifted upward. This is because of the fact that the $I M$ value assumed in the Ludescher (2003) model for single, light trucks ( $I M=0.4$ for total static live load up to $300 \mathrm{kN}$ ) is considerably higher than the code-specified $I M$ value.
One might conclude from the variable $I M$ results that the effects of simultaneous crossings are negligible — in particular, for $m=3.0$ but that the basic $\gamma$ factors in the North American codes are too low. It should be noted, however, that the $I M$ value for single, light trucks assumed in the Ludescher (2003) model represents an estimate of the expected value that is on the high side compared with other values reported in the literature (e.g., Bailey 1996; Lee et al. 2006). If this model were modified by reducing this value (e.g., to 0.15 or 0.25 ), it is 
expected that the results would fall between the constant and variable $I M$ results presented in Fig. 10.

To further study the influence of the DLA on $\gamma_{2}$, it is believed that a multidegree of freedom dynamic modeling approach (see Ludescher 2003; Lee et al. 2006) would be appropriate, with consideration given to uncertainties in the road surface quality and other significant parameters. While the approach used herein does not consider all of these parameters, it has the advantage of being conservative (in that it may slightly overestimate simultaneous crossing effects) and not dependent on numerous site-specific parameters.

\section{Conclusions}

Based on the work presented in this paper, the following conclusions are drawn:

- The bridge code procedures described in this paper for fatigue design all employ approximate approaches for relating the fatigue damage because of real truck traffic to a design stress range. In general, these approaches lead to a high degree of scatter because of variations in the influence line shape and bridge span. In addition, none of the described codes considers the effects of simultaneous vehicle crossings in the calculation of fatigue damage.

- The effects of simultaneous vehicle crossings on the damage equivalence factors, $\gamma$, for North American roadway bridges can be considerable for certain traffic conditions, influence lines, spans, and cross sections. Specifically, long-span single box girder bridges can be significantly influenced by these effects-in particular, at interior supports where the p2tr-a influence line applies and under bidirectional traffic.

- A simulation-based study has shown that the amplification factor $\left(\gamma_{2}\right)$ for considering these effects can be as high as $\sim 1.2$ for steel girders (that is, with $m=3.0$ ), if average values for the different influence lines are compared at a truck flow rate of 0.2 vehicles $/ \mathrm{s}$ and a constant $I M$ model is assumed. For the case of $m=6.85$, this value increases to 1.54 . This increase can be explained by the fact that the large cycles because of simultaneous crossings do more damage with the flatter S-N curve slope. If a variable $I M$ model is assumed, then the effects of simultaneous crossings on fatigue damage will generally be reduced.

- Eqs. (11) and (12) are proposed for amplifying the damage equivalence factor in cases where the effects of simultaneous vehicle crossings are expected to be significant. Along with this equation, a set of effective simultaneous crossing rates, ranging from 0 to $13.5 \%$, are proposed for calculating this amplification factor.

Further study would be of interest to extend these results to bridges supporting more than two lanes of traffic. It is believed that the presented results effectively bound the problem. However, additional simulations are recommended to quantify the effects of simultaneous crossings for structures with intermediate degrees of torsional stiffness (that is, between single box girder and multigirder bridges), as well as unidirectional traffic with a higher percentage of trucks in lane 1 (that is, the slow or far right-hand lane). Simulations wherein the truck flow rates are allowed to vary, to reflect daily, weekly, or seasonal trends, are also recommended. Last, it is suggested that a similar simulation-based approach be employed to investigate the effects of simultaneous vehicle crossings on the design factors used in the infinite-life domain.

\section{Acknowledgments}

Technical advice concerning this research provided by Thierry Meystre, Professor Bruce Hellinga (University of Waterloo), and Reza Noroozisanani is gratefully acknowledged.

\section{References}

AASHTO. (2008). "AASHTO LRFD bridge design specification: Fourth edition with 2008 interim revisions," Washington, DC.

Aluminum Association. (2010). ADM-10: Aluminum design manualSpecifications and guidelines for aluminum structures, Arlington, VA.

Bailey, S. F. (1996). "Basic principles and load models for the structural safety evaluation of existing road bridges." Doctoral thesis No. 1467, École Polytechnique Fédérale de Lausanne, Lausanne, Switzerland.

Canadian Standards Association (CSA). (2007). "Calibration report for CAN/CSA-S6-06: Canadian highway bridge design code." Mississauga, ON, Canada.

Canadian Standards Association (CSA). (2011a). "CAN/CSA-S6-06: Canadian highway bridge design code (including supplement No. 2 revisions)." Mississauga, ON, Canada.

Canadian Standards Association (CSA). (2011b). "Commentary on CAN/ CSA-S6-06: Canadian highway bridge design code (including supplement No. 2 revisions)." Mississauga, ON, Canada.

Coughlin, R., and Walbridge, S. (2011). "Fatigue correction factors for welded aluminum highway structures." Can. J. Civ. Eng., 38(10), 1082-1091.

European Committee for Standardization (CEN). (2002). EN 1991-2: Actions on structures, part 2: Traffic loads on bridges, Brussels, Belgium.

European Committee for Standardization (CEN). (2005). EN 1993-1-9: Design of steel structures, part 1-9: Fatigue, Brussels, Belgium.

European Committee for Standardization (CEN). (2006). EN 1993-2: Design of steel structures, part 2: Steel bridges, Brussels, Belgium.

Fischer, V. (2012). "Effect of simultaneous vehicle crossings on the North American fatigue correction factors." Master's thesis report, École Polytechnique Fédérale de Lausanne, Lausanne, Switzerland 〈http: //infoscience.epfl.ch/record/182820 $\rangle$.

Hirt, M. A., Bez, R., and Nussbaumer, A. (2006). Traite de génie civil, vol. 10: Construction métallique-Notions fondamentales et méthodes de dimensionnement. Presses Polytechniques et Universitaires Romandes, Lausanne, Switzerland.

Lee, H.-H., Jeon, J.-C., Kyung, K.-S., and Mori, T. (2006). "Influence of moving vehicle on fatigue of steel girder bridge." Int. J. Steel Struct., 6(4), 269-278.

Ludescher, H. (2003). "Berücksichtigung von dyna-mischern Verkehrslasten beim Tragsicherheits-nachweis von Strassenbrücken." Doctoral thesis No. 2894, École Polytechnique Fédérale de Lausanne, Lausanne, Switzerland.

Maclean, D. I., and Marsh, M. L. (1998). "Synthesis of highway practice 266: dynamic impact factors for bridges." Rep. 266, National Cooperative Highway Research Program, Washington, DC.

Maddah, N., and Nussbaumer, A. (2012). "Evaluation of Eurocode damage equivalent factor based on traffic simulation." Proc., 6th Int. Conf. on Bridge Maintenance, Safety, and Management, F. Biodini and D. M. Frangopol, eds., Taylor \& Francis, London.

Meystre, T., and Hirt, M. A. (2006). Evaluation de ponts routiers existants avec un modèle de charge de trafic actualisé, Office Fédéral des Routes, Bern, Switzerland.

Moses, F., Schilling, C. G., and Raju, K. S. (1987). "Fatigue evaluation procedures for steel bridges." Rep. 299, National Cooperative Highway Research Program, Washington, DC.

Nowak, A. S. (1993). "Live load model for highway bridges." Struct. Saf., 13(1-2), 53-66.

O’Brien, E. J., and Enright, B. (2011). "Modeling same-direction two-lane traffic for bridge loading." Struct. Saf., 33(4-5), 296-304.

Snyder, R. E., Likins, G. E., and Moses, F. (1985). "Loading Spectrum Experience by Bridge Structures in the United States." Rep. FHWA/RD 85/012, Federal Highway Administration, Cleveland, OH.

Swiss Society of Engineers and Architects (SIA). (2003a). "Actions on structures." SIA 261, Zurich, Switzerland.

Swiss Society of Engineers and Architects (SIA). (2003b). "Steel Construction." SIA 263, Zurich, Switzerland.

Transportation Research Board (TRB). (2010). Highway capacity manual 2010, National Research Council, Washington, DC. 